\title{
Analytic Discs Attached to Manifolds with Boundary
}

\author{
By \\ Luca BARACCO* and Giuseppe ZAMPIERI*
}

\section{§1. Introduction}

Let $X$ be a complex manifold of dimension $n, M^{+}$a closed half-space with boundary $M, A$ an analytic disc of $X$ "attached" to $M^{+}$, tangent to $M$ at some point $z_{0}$ of $\partial A \cap M$, and intersecting $\dot{M}^{+}$in any neighbourhood of $z_{0}$. Then holomorphic functions extend from $\dot{M}^{+}$to a full neighborhood of $z_{0}$. This theorem refines the results of [1] where the boundary $\partial A$ (instead of the whole $A$ ) was supposed to intersect $\dot{M}^{+}$. The argument of the proof consists in constructing a (closed) manifold with boundary $W$, contained in the envelop of holomorphy of $\dot{M}^{+}$and such that $A \subset W$ but $A \not \subset \partial W$. In this situation it is easy to find a new small $\operatorname{disc} A_{1} \subset A$ with $\partial A_{1} \not \subset \partial W$. We are therefore in a situation similar to [1], and get the conclusion by exhibiting a disc transversal to $\partial W$ at $z_{0}$.

Extension of holomorphic functions by the aid of tangent discs attached to $M$ and of "defect 0 " is a particular case of a general theorem of "wedge extendibility" of CR-functions by A. Tumanov; the new part of our theorem is that no assumptions on "defect" are made.

This paper is tightly inspired to the results and the techniques by $\mathrm{A}$. Tumanov [7]. We also owe to A. Tumanov a great help during private communications.

$\S 2$.

Let $X$ be a complex manifold of dimension $n, M$ a real submanifold of

Communicated by K. Saito, January 6, 1997.

1991 Mathematics Subject Classification(s): 32

* Department of Math., Univ. of Padova, V. Belzoni 7, 35131 Padova, Italy 
$X, M^{+}$one of the two closed half-spaces with boundary $M, A=A(\tau), \tau \in \Delta$ an analytic disc of $X, z_{0}=A(1)$ a point of $\partial A \cap M,\{B\}$ the system of spheres of center $z_{0}$. Let $C^{k, \alpha}$ be the functions whose derivatives up to the order $k$ are $\alpha$-Lipschitz continuous. We assume $M$ to be $C^{2, \alpha}$ and $A$ to be $C^{1, \alpha}$ up to the boundary and small. We recall the result by [1].

Theorem A。 ([1, Theorem 1]) Assume

$$
\partial_{\tau} A(1) \in T_{z_{0}}^{C} M
$$

$$
\partial A \subset M^{+}
$$

$$
\text { there exists } z_{1} \in \partial A \text { with } z_{1} \in \stackrel{M}{ }^{+} \text {. }
$$

Then for any $B \supset A$ there is $B^{\prime} \subset B$ such that holomorphic functions extend from $\dot{M}^{+}$to $B^{\prime}$.

It is essential in the previous statement that $z_{1} \in \partial A$ (in addition to $\left.z_{1} \in \stackrel{\circ}{ }^{+}\right)$. As for the case $z_{1} \in \operatorname{int} A$, we can reduce to the former case when we strengthen (ii) to " $A \subset M^{+}$". In this case extension from $B \cap M^{+}$to a suitable $B^{\prime}$ holds provided that $B$ contains $z_{1}$ ([1, Corollary 4$]$ ).

What happens when $z_{1} \notin \partial A$ and $A \not \subset M^{+}$? Holomorphic extension seems not to take place. However it holds when a sequence converging to $z_{0}$ of such points $z_{1} \notin \partial A$ does exist.

Theorem 1. Let $M$ be $C^{3, \alpha}$, and assume that $\partial A$ is a $C^{2, \alpha}$ curve with $A(1)=z_{0}$. Suppose

(i) $\partial_{\tau} A(1) \in T_{z_{0}}^{C} M$

(ii) $\partial A \subset M^{+}$

(iii) $A \cap \dot{M}^{+} \cap B^{\prime} \neq \emptyset \forall B^{\prime}$.

Then if $B \supset A$ there exists $B^{\prime}$ such that any holomorphic function extends from $B \cap \dot{M}^{+}$to $B^{\prime}$.

Proof. When $\partial A \not \subset M$, the statement is the same as in Theorem A. For completeness, we shall treat it at the end of the proof. Assume therefore $\partial A \subset M$.

(a) Construction of a manifold with boundary $W$ such that $W \supset A$. We 
assume $M$ is described by $y_{1}=h\left(x_{1}, z^{\prime}\right), z^{\prime} \in C^{n-1}=T_{z_{0}}^{C} M, h(0,0)=0, \partial h(0,0)=0$. Set $v_{2}=i \partial_{\tau} A(1)\left(\in T_{z_{0}}^{C} M\right)$, choose $v_{1} \in T_{z_{0}} M$ transversal to $T_{z_{0}}^{C} M$, e.g. $v_{1}=e_{1}$, and write:

$$
T_{z_{0}} M=\boldsymbol{R} v_{1} \oplus \boldsymbol{C} v_{2} \oplus \boldsymbol{C}^{n-2} .
$$

Let $w^{\prime}=z^{\prime} \circ A$ take $w_{0}^{\prime \prime} \in C^{n-2}, s \in \mathbb{R}$ and consider the equation

$$
u(\tau)=-T_{1}\left(h\left(u(\tau), w^{\prime}(\tau)+\left(0, w_{0}^{\prime \prime}\right)\right)+s \quad \tau \in \partial \Delta\right.
$$

in the unknown $u$. Note that for $s=0, w_{0}^{\prime \prime}=0$, the " $x_{1}$-component" $u$ of $A$ satisfies (1). We need the following technical tool.

Lemma 2. Let $h$ be $C^{k . \alpha}$. Then for any $w^{\prime}$ in $C^{k-1 . \alpha}$ small, there is an unique solution to $(1) u(\tau)=u_{s, w_{0}^{\prime \prime}}(\tau), \tau \in \partial \Delta$, which belongs to $\left.C^{k-1, \alpha}\right|_{\tau, s, w_{0}^{\prime \prime}}$.

Proof. For $j \leq k-1$, let

$$
\begin{gathered}
F: C^{j, \alpha}(\partial \Delta, \boldsymbol{R}) \times C^{n-2} \times \boldsymbol{R} \rightarrow C^{j, \alpha}(\partial \Delta, \boldsymbol{R}) \\
\left(u, w_{0}^{\prime \prime}, s\right) \mapsto u+T_{1}\left(h\left(u, w^{\prime}+\left(0, w_{0}^{\prime \prime}\right)\right)\right)-s .
\end{gathered}
$$

Let $v=h\left(u, w^{\prime}+\left(0, w_{0}^{\prime \prime}\right)\right)$ in $\partial \Delta$, and define

$$
D_{w_{0}^{\prime \prime}, s}=\left(u+i v, w^{\prime}+\left(0, w_{0}^{\prime \prime}\right)\right) \text { in } \partial \Delta .
$$

We have:

$$
D_{w_{0}^{\prime \prime} . s} \text { extends holomorphically to } \Delta \text { with } D_{w_{0}^{\prime \prime} \cdot s}(1)=s
$$

if and only if

$$
F=0 \text {. }
$$

If $h$ is $C^{k, \alpha}$, then $F$ is $C^{1}$ (as application between functional spaces) and we have

$$
F^{\prime}\left(\dot{u}, w_{0}^{\prime \prime}, s\right)=\dot{u}+T_{1}\left(\partial_{x} h \dot{u}+\partial_{w^{\prime \prime}} h w_{0}^{\prime \prime}+\partial_{\bar{w}^{\prime}}, h \bar{w}^{\prime \prime}\right)-s
$$

We have

$$
\left\{\begin{array}{l}
F(0,0,0)=0 \\
F^{\prime}(\dot{u})=\dot{u}+T_{1}\left(\partial_{x} h \dot{u}\right) .
\end{array}\right.
$$

Thus the equation $F=0$ has solution $u$ in $C^{j \cdot \alpha}(\partial \Delta, R)$ (and this depends $C^{k-1-j, \alpha}$ 
on data $w, x, \zeta$ and on $w_{0}^{\prime \prime}, s$. (Cf. [5])).

Q.E.D.

In particular, since $h$ is $C^{3, \alpha}$ and $A$ is $C^{2, \alpha}$ up to the boundary, then setting $w^{\prime}=z^{\prime} \circ A$, (1) has an unique solution

$$
u=\left.u_{w_{0}^{\prime \prime}, s}(\tau) \in C^{2, \alpha}\right|_{\tau, w_{0}^{\prime \prime}, s} .
$$

With $v=T_{1}(u)+h\left(s, w_{0}\right)$, define $D=D_{w_{0}^{\prime \prime}, s}$ as in the proof of Lemma 2. Clearly for $s=0$ and $w_{0}^{\prime \prime}=0, D$ equals the initial disc $A$. Note also that for any $s$ and $w_{0}^{\prime \prime}, D$ is attached to $M$.

We have that

$$
\begin{aligned}
D: C_{w_{0}^{\prime \prime}}^{n-2} \times \Delta_{\tau} \times \boldsymbol{R}_{s} & \rightarrow C^{n} \\
\left(w_{0}^{\prime \prime}, \tau, s\right) & \mapsto D_{w_{0}^{\prime \prime} . s}(\tau)
\end{aligned}
$$

verifies

$$
\operatorname{rank}^{R} \partial_{w_{0}^{\prime \prime} \tau, s} D=2 n-1
$$

and therefore the range of $D$ is a manifold $S$ of class $C^{1, \alpha}$ with boundary $\partial S=D\left(C^{n-2} \times \partial \Delta_{\tau} \times \boldsymbol{R}_{s}\right)$ in a neighborhood of $z_{0}=D_{0,0}(1)$. Note that $\partial S$ is generic and $\partial S \subset M$. Note also that $A \subset S$. Define

$$
W=\bigcup_{\zeta_{1}}-i e_{1} \zeta_{1}+S
$$

It is clear that any holomorphic function extends from $\stackrel{\circ}{ }^{+}$to $W \cup \dot{M}^{+}$. In a neighborhood $B_{1}$ of $z_{0}=0$, possibly smaller than $B$, we can describe $W$ by:

$$
y_{1}=g\left(x_{1}, x_{2}+i \zeta_{2}, z^{\prime \prime}\right)+\zeta_{1} \quad \zeta_{1} \leq 0, \zeta_{2} \leq 0,
$$

and

$$
S: W \cap\left\{\zeta_{1}=0\right\}
$$

(b) Perturbation of $W$ and of $A$ such that $\partial A \not \subset W$. We perturb $g$ for $\zeta_{2}>\varepsilon$ to a new function $\tilde{g} \geq g$ such that $\left\{y_{1}<\tilde{g}\right\} \subset W \cup \dot{M}^{+}$and such that the initial disc $A=(u+i v, w)$ verifies in a point $\tau_{1} \in \Delta$ :

$$
v\left(\tau_{1}\right)<\tilde{g}\left(u\left(\tau_{1}, w\left(\tau_{1}\right)\right)\right.
$$

We also suppose $z_{1}=A\left(\tau_{1}\right)$ close to $z_{0}$. Let us still write $g$ instead of $\tilde{g}$ and 
denote by $S$ and $W$ the manifolds with boundary defined by (2) and (3) for this new $g$. Thus (4) is equivalent to

$$
z_{1} \in A \cap \stackrel{\circ}{W}
$$

Take $\tilde{\Delta} \subset \Delta$ such that $\tau_{1} \in \partial \tilde{\Delta}$ and $\partial \tilde{\Delta}=\partial \Delta$ at 1 . Set $\tilde{A}=A \circ \Phi$ where $\Phi$ is an analytic diffeomorphism $\Delta \underset{\oplus}{\rightarrow} \tilde{\Delta}$. Let us write (2) as

$$
\left\{\begin{array}{l}
y_{1}=g\left(x_{1}, x_{2}, z^{\prime \prime}\right)+\zeta_{1} \\
y_{2}=\zeta_{2}
\end{array}\right.
$$

with $\zeta_{1}, \zeta_{2} \leq 0$. In these coordinates $S$ is defined by $\zeta_{1}=0$ and $\partial S$ by $\zeta_{1}=\zeta_{2}=0$. Note that $\partial \tilde{A}=\partial A$ at $z_{0}$ and that $\partial A \subset \partial S$; it follows that $\zeta_{1}=0$, $\zeta_{2}=0$ at $\tau=1$. We shall now use only the new disc $\tilde{A}$, and call again $A$.

(c) Construction of a transversal disc. Let $\zeta_{1}(\tau), \zeta_{2}(\tau)$ be defined by (5) over $A_{1}$. Consider the system:

$$
\begin{cases}u_{1}=-T_{1}\left(g\left(u_{1}(\tau), u_{2}(\tau)+i \zeta_{2}(\tau), w^{\prime \prime}(\tau)\right)+(1-\eta) \zeta_{1}(\tau)\right), & \tau=e^{i \theta} \\ u_{2}(\tau)=-T_{1}\left(\zeta_{2}(\tau)\right), & \tau=e^{i \theta}\end{cases}
$$

There exists an unique solution $\left(u_{1}, u_{2}\right)=\left(u_{1 \eta}, u_{2 \eta}\right)$ in $\left.C^{1, \alpha}\right|_{\theta}$. Moreover if we set $v_{1}=T_{1}\left(u_{1}\right), v_{2}=T_{1}\left(u_{2}\right)$, and

$$
A_{\eta}=\left(u_{1 \eta}+i v_{1 \eta}, u_{2 \eta}+i v_{2 \eta}, w^{\prime \prime}\right)
$$

we have that

$$
\partial_{\tau} A_{\eta} \quad \text { is }\left.\quad C^{1}\right|_{\eta} .
$$

In fact $\partial_{\eta} u_{2 \eta} \equiv 0$, whereas $\partial_{\eta} u_{1 \eta}$ is a solution of:

$$
\partial_{\eta} u_{1 \eta}+T_{1}\left(\partial_{x_{1}} g \partial_{\eta} u_{1 \eta}-\zeta_{1}\right)=0 .
$$

Since $\partial g$ and $\zeta_{1}$ are $C^{2, \alpha}$, then $\partial_{\eta} u_{1 \eta}$ is $C^{1, \alpha}(\partial \Delta, R)$; in particular, with $\tau=e^{i \theta}$, $\partial_{\theta} \partial_{\eta} u_{1 \eta}$ exists and is continuous.

As for $\partial_{\theta} \partial_{\eta} v_{1 \eta}$, we begin by remarking that

$$
v_{1 \eta}=g\left(u_{1 \eta}, u_{2}+i \zeta_{2}, w^{\prime \prime}\right)+(1-\eta) \zeta_{1} \text { on } \partial \Delta .
$$

It follows that 


$$
\partial_{\eta} v_{1 \eta}=\partial_{x_{1}} g\left(u_{1 \eta}, w\right) \partial_{\eta} u_{\eta}-\zeta \text { belongs to } C^{1, \alpha}(\partial \Delta, \boldsymbol{R})
$$

We derive with respect to $\theta$ and obtain:

$$
\begin{aligned}
\partial_{\theta} \partial_{\eta} v_{1 \eta} & =\partial_{\theta}\left(\partial_{x_{1}} g\left(u_{1 \eta}, w\right) \partial_{\eta} u_{\eta}-\zeta\right) \\
& =\partial_{x_{1}}^{2} g \partial_{\theta} u_{1 \eta} \partial_{\eta} u_{1 \eta}+\partial_{x_{1}} g \partial_{\theta} \partial_{\eta} u_{1 \eta}-\partial_{\theta} \zeta_{1} .
\end{aligned}
$$

It is easy to check that all the terms on the right have at least class $C^{0}$; thus also $\partial_{\theta} \partial_{\eta} v_{1 \eta}$ is $C^{0}$. Finally Cauchy-Riemann equations yield:

$$
\partial_{\theta}\left(u_{1 \eta}+i v_{1 \eta}\right)=i e^{i \theta} \partial_{\tau}\left(u_{1 \eta}+i v_{1 \eta}\right)
$$

and we are done. It follows from (7):

$$
\partial_{\tau} A_{\eta}=\partial_{\tau} A+\left.\eta \partial_{\tau} \partial_{\eta} A_{\eta}\right|_{\eta=0}+o(\eta)
$$

Set $r=y_{1}-g$. We prove now that

$$
\left.\mathfrak{R e}\left\langle\partial r \circ A, \partial_{\tau} \partial_{\eta} A_{\eta}\right\rangle\right|_{\tau=1}<0 .
$$

In fact one finds a real function $\lambda$ on $\partial \Delta$ such that $\lambda \partial r \circ A$ extends holomorphically to $\Delta$ and notice that then:

$$
\left\langle\lambda \partial r \circ A, \partial_{\eta} A_{\eta}\right\rangle=\left\langle\lambda \partial_{1} r \circ A, \partial_{\eta} A_{\eta}\right\rangle
$$

is a holomorphic function. The real part $\varphi$ of this holomorphic function verifies $\left.\varphi\right|_{\partial \Delta}=-\zeta \lambda$ whence: $\left.\varphi\right|_{\partial \Delta} \geq 0, \varphi(1)=0, \varphi\left(\tau_{1}\right)>0$. (Here $\tau_{1}$ is such that $A\left(\tau_{1}\right)=z_{1}$.) Then Hopf Lemma implies (9). Plugging together (8) and (9) we get

$$
\mathfrak{R e}\left\langle\partial r, \partial_{\tau} A_{\eta}\right\rangle(1)<0 .
$$

(d) Construction of a dihedron. We denote again by $\zeta_{1}, \zeta_{2}, w^{\prime \prime}$ the components of this new $\operatorname{disc} A_{\eta}$ and solve the equations:

$$
\left\{\begin{array}{l}
u_{1}=-T_{1}\left(g\left(u_{1}(\tau), u_{2}(\tau)+i \zeta_{2}(\tau), w^{\prime \prime}(\tau)+w_{0}^{\prime \prime}\right)+\zeta_{1}(\tau)\right)+s \\
u_{2}=-T_{1}\left(\zeta_{2}(\tau)\right) \\
v_{1}=-T_{1} u_{1}+g\left(s, 0, w_{0}^{\prime \prime}\right) \\
v_{2}=T_{1}\left(u_{2}\right) .
\end{array}\right.
$$

Let $A=A_{s, w_{0}^{\prime \prime}}$ be defined by (11). Note that 


$$
\left\{\begin{array}{l}
\partial A \subset W \text { and } \partial A \subset S \text { at } z_{0} \\
A \text { is transversal to } M \text { (for } s, w^{\prime \prime} \text { small). }
\end{array}\right.
$$

Define

$$
S_{1}=\left\{A_{s, w_{0}^{\prime \prime}}(\tau) ; \tau \in \Delta, s \text { and } w_{0}^{\prime \prime} \in \boldsymbol{R} \times \boldsymbol{C}^{n-2}\right\}
$$

We have

$$
\left\{\begin{array}{l}
\partial S_{1} \text { generic } \\
\left.\partial S_{1}=\partial S \text { at } z_{0} \text { (hence } \partial S_{1} \subset M\right)
\end{array}\right.
$$

where the latter of (14) follows from the former of (12). By using $S_{1}$ and $M$ one gets a dihedron $V$ with non-proper tangent cone, such that any $f$ holomorphic on $\stackrel{\circ}{M}^{+} \cap B$, for $B \supset A$, is extended to $V$ at $z_{0}$.

(e) Conclusion of the proof in the case $\partial A \subset M$. The dihedron $V$ has generic edge $\partial S_{1}$. We approximate $V$ by an increasing sequence $V_{v}$ of domains with $C^{2}$ boundary such that

$$
V_{v} \subset V, \partial V_{v} \supset \partial S_{1} \quad \forall v .
$$

It is obvious that for large $v, V_{v}$ has at least one Levi-pseudoconcavity. Then germs of holomorphic functions extends from $V_{v}$ to a full neighborhood of $z_{0}$ (according to a famous theorem by Hans Lewy).

(f) The case $\partial A \not \subset M$ (cf. [1]). In this case the proof is simpler. Let $M$ be defined by $y_{1}=h\left(x, z^{\prime}\right) \quad\left(z^{\prime}=\left(z_{2}, z_{3}, \cdots\right)\right)$ with $h(0)=0, \partial h(0)=0$, write $A=\left(u+i v, w^{\prime}\right)$, and put $\zeta_{1}=v-(h \circ A)$. Solve

$$
u_{\eta}=-T_{1}\left(h \left(u_{\eta}(\tau), w^{\prime}(\tau)+(1-\eta)\left(\zeta_{1}(\tau)\right)\right.\right.
$$

This produces a family of discs $A_{\eta}=\left(u_{\eta}+i v_{\eta}, w^{\prime}\right)\left(v_{\eta}=T_{1}\left(u_{\eta}\right)\right)$ which verify by the same argument as above:

$$
\mathfrak{R e}<\partial r \circ A, \partial_{\tau} A_{\eta}>\left.\right|_{\tau=1}<0
$$

for any sufficiently small $\eta$. Let $\zeta_{1}, w^{\prime}$ still denote the components of $A_{\eta}$ and solve

$$
u(\tau)=-T_{1}\left(h\left(u(\tau), z^{\prime}(\tau)+w_{0}^{\prime}\right)+\zeta_{1}(\tau)\right)+s .
$$


By taking the union of the discs $A_{s, w_{0}^{\prime}}(\tau)$ one gets a manifold $S_{1}$ such that $\partial S_{1} \subset M^{+}$and $S_{1} \cap M$ is a generic manifold. We thus get a dihedron $V$ with edge $S_{1} \cap M$ and such that functions extend from $\dot{M}^{+} \cap B$ to $V$ at $z_{0}$ $(B \supset A)$. The conclusion is the same as above. $\quad$ Q.E.D.

\section{References}

[1] Baracco, L. and Zampieri, G., Analytic discs attached to half-spaces of $\boldsymbol{C}^{n}$ and extension of holomorphic functions, Preprint (1996).

[2] Boggess, A., CR Manifolds and the tangential Cauchy-Riemann complex, Stud. Adv. Math. CRC Press (1991).

[3] Baouendi, M. S., Rothshild, L. P. and Trepreau, J. M., On the geometry of analytic discs attached to real manifolds, J. Diff. Geom., 39 (1994), 379-405.

[4] Trepreau, J. M., Sur le prolongement holomorphe des fonctions C-R définies sur une hypersurface réelle de classe $C^{2}$ dans $C^{n}$, Invent. Math., 83 (1986), 583-592.

[5] Tumanov, A., Connections and propagation of analyticity for CR functions, Duke Math. J., 731 (1994), 1-24.

[6] On the propagation of extendibility of $C R$ functions, "Complex Analysis and Geometry”, Lect. Notes in Math., Marcel-Dekker (1995), 479-498.

[7] - Extending CR functions from manifolds with boundaries, Math. Res. Letters, 2 (1995), 629-642. 